\title{
Inspecting Management Strategies of Hepatocellular Carcinoma in a Tertiary Centre in Western Rajasthan
}

\author{
Ganaraj Kulamarva ${ }^{1}$, Supriya Prathibha Shankaranarayana Bhat ${ }^{2}$, \\ Sunil Dadhich ${ }^{3}$, Narendra Bhargava ${ }^{4}$, Prabhat Ranjan ${ }^{5}$ \\ 1, 2 Department of General Medicine, K.S. Hegde Medical Academy, Deralakatte, Mangaluru, Karnataka, India. \\ 3, 4 Department of Gastroenterology, Dr. Sampurnanand Medical College, Jodhpur, Western Rajasthan, India. \\ ${ }^{5}$ Department of Gastroenterology, Basu Nursing Home, Siliguri, West Bengal, India.
}

\section{ABSTRACT}

\section{BACKGROUND}

Hepatocellular carcinoma (HCC) is a lethal malignancy which mostly develops in patients with cirrhosis. It is usually diagnosed late in the course of the illness and the median survival following diagnosis ranges between 6 - 20 months. India lacks data on management strategies and their efficacy. In the absence of data on treatment protocols and its adequacy; we evaluated our own centre data for a period of 1 year to get the estimate of incidence, aetiology, treatment adequacy and response to treatment. Barcelona Clinic Liver Cancer (BCLC) prognostic staging classification comprising five stages is used for prognostication, which is based on the extent of the primary lesion, performance status, vascular invasion and extrahepatic spread. Surgical therapies including resection and transplantation are feasible in early stages (BCLC stage 0 and stage A). Trans arterial chemoembolisation is recommended in intermediate stage (BCLC stage B) while systemic therapies are recommended in advanced stage (BCLC stage $C$ ). Best supportive care is recommended in terminal stage (BCLC stage D). This study has included BCLC staging for staging classification and patients were assessed for adequacy of management.

\section{METHODS}

This study was done as a retrospective hospital based observational study. All HCC patients presenting to Mahatma Gandhi Hospital attached to Dr. Sampurnanand Medical College, Jodhpur, Western Rajasthan from January to December 2014 were included. HCC was diagnosed based on European Association for the study of the Liver-European Organisation for Research and Treatment of Cancer (EASL-EORTC) clinical practice guidelines 2011. Patients were classified according to Barcelona Clinic Liver Cancer staging and management given was recorded.

\section{RESULTS}

Thirty-two patients who were diagnosed with HCC between January to December 2014 were included in the study. In three fourths of the patients (24) HCC was diagnosed based on typical findings on dynamic imaging studies (triple phase contrast enhanced CT-computed tomography abdomen and / or MRI- magnetic resonance imaging abdomen). Liver biopsy was needed in one fourth of the patients. Majority of the patients (87.5\%) had cirrhosis of the liver at the time of diagnosis of HCC. Some of these patients [5 (17.8\%)] were known cirrhotic patients.

\section{CONCLUSIONS}

Hepatitis B was the most common aetiology of HCC as mentioned previously in other studies, which is vaccine preventable. HCC is rarely diagnosed at an early stage in developing countries. Various treatment modalities are available which depend on the stage, local expertise and affordability. If the surveillance recommendations are strictly adhered, HCC can be diagnosed at an early stage. Affordability and compliance will remain issues in HCC management in our country increasing the socio-economic burden on the society.

\section{KEY WORDS}

Hepatocellular Carcinoma (HCC), BCLC Staging, Survival
Corresponding Author: Dr. Supriya P.S., 4 C, Shanthiniketan staff quarters, K.S. Hegde Medical Academy, Deralakatte, Mangaluru - 575018, Karnataka, India. E-mail:drsupriyaps@gmail.com

DOI: $10.14260 /$ jemds/2021/277

How to Cite This Article: Kulamarva G, Bhat SP, Dadhich S, et al. Inspecting management strategies of hepatocellular carcinoma in a tertiary centre in Western Rajasthan. J Evolution Med Dent Sci 2021;10(18):1314-1318, DOI: 10.14260/jemds/2021/277

Submission 18-09-2020,

Peer Review 05-03-2021,

Acceptance 12-03-2021,

Published 03-05-2021.

Copyright (C) 2021 Ganaraj Kulamarva et al. This is an open access article distributed under Creative Commons Attribution License [Attribution 4.0 International (CC BY 4.0)] 


\section{BACKGROUND}

Hepatocellular carcinoma is a lethal malignancy that mostly develops in patients with cirrhosis. It is usually diagnosed late in the course of the illness and the median survival following diagnosis ranges between 6 - 20 months. ${ }^{1}$ BCLC prognostic staging classification comprising five stages is used for prognostication, which is based on the extent of the primary lesion, performance status, vascular invasion and extrahepatic spread.2,3 Early diagnosis and management could bring down the mortality associated with hepatocellular carcinoma significantly. HCC is diagnosed based on European Association for the study of the Liver-European Organisation for Research and Treatment of Cancer clinical practice guidelines 2011.4 Approximately 7.5 lakhs new cases of HCC are diagnosed per year in the entire world making it the 5 th common cause of cancers in human beings. Overall, 7 lakh deaths every year are due to HCC making it the $3^{\text {rd }}$ highest cancer responsible for death in human beings. ${ }^{5}$ Patients with advanced unresectable HCC have a dismal prognosis with median survival rates as low as $2-3$ months despite receiving best supportive care (BSC). Sorafenib is an oral multikinase inhibitor and the only drug till 2017 which had demonstrated a survival benefit over BSC in advanced HCC. Diarrhoea and dermatological side effects are seen in about $40 \%$ of patients on sorafenib and may require dose modification or drug discontinuation. Drug discontinuation was needed in up to one fifth of the patients who were included in the global investigation of therapeutic decisions in hepatocellular carcinoma and of its treatment with sorafenib (GIDEON) observational cohort study. Till 2017 there were no other second line treatment options for patients with advanced HCC who failed to respond to sorafenib. Recently other kinase inhibitors like lenvatinib, regorafenib and check point inhibitors like nivolumab and pembrolizumab have been approved for use in HCC. ${ }^{6}$ Lenvatinib has been approved as a first line agent for HCC while others have been approved as second line agents for HCC. Resection surgery is feasible in BCLC stage 0 and stage A patients but is associated with recurrence rates of $10 \%$ per year. Ideal candidates for liver resection have solitary tumours with good liver function, hepatic vein to portal system gradient $\leq 10 \mathrm{~mm} \mathrm{Hg}$ or platelet count $\geq 10000 /$ microlitre. Recurrence rates at 5 years are as high as 70 to $80 \% .{ }^{5}$ Liver transplantation is feasible in BCLC stage B patients satisfying Milan criteria. Patients beyond the Milan criteria who respond successfully to down staging therapies and come within Milan criteria have also been considered for liver transplantation. University of California San Francisco (UCSF) criteria which generated more potential recipients compared to Milan criteria has claimed similar post liver transplant survival. ${ }^{6}$ Scarcity of liver donors, significant time spent waiting for a suitable donor and chance of disease progression during the waiting period decrease the number of liver transplantation surgeries done in HCC. ${ }^{7}$ Trans arterial chemo embolisation (TACE) is recommended in BCLC stage $\mathrm{C}$ patients. It exploits the fact that HCC typically receives its blood supply from the hepatic artery or its branches. In TACE the tumour feeding blood vessels are infused with a cytotoxic agent followed by an embolisation agent. This causes a strong cytotoxic and ischemic effect at the tumour site. TACE can be done with chemotherapy emulsion with lipiodol called conventional TACE or with drug eluting beads (TACE-DEB). Subsequent sessions of TACE are required in patients with residual viable HCC on follow up contrast enhanced imaging. Complications of TACE include ischemia at nontumorous areas presenting as cholecystitis, pulmonary embolism, hepatic abscess and gastric mucosal injury. ${ }^{8}$ Ablation therapies are recommended in patients with BCLC stage 0 and stage A tumours which are not suitable for surgical therapy. Percutaneous ethanol injection was the first ablative therapy used in HCC.

Thermal ablative therapies have replaced ethanol injection. Radiofrequency ablation, microwave ablation, laser ablation and cryoablation are various thermal ablative therapies used in HCC treatment. ${ }^{6}$

India highly lacks data on management strategies and its survival benefits. In the absence of data on treatment protocols and its adequacy; we evaluated our own centre data for a period of 1 year to get estimate of incidence, aetiology, treatment adequacy and response to treatment.

\section{Objectives}

1. To estimate the risk factors of HCC.

2. To assess the BCLC staging specific management strategies of HCC management at our centre with regard to diagnosis and treatment.

\section{METHODS}

This was a retrospective hospital based single centre observational study of consecutive diagnosed patients with hepatocellular carcinoma (HCC) presenting to gastroenterology department of Mahatma Gandhi Hospital attached to Dr SN Medical College Jodhpur from January to December 2014. The study was approved by ethics committee. HCC was diagnosed based on EASL-EORTC clinical practice guidelines 2012.4 Patients case sheets were seen to see their Barcelona Clinic Liver Cancer stage and management given was noted. Stage appropriate treatment was liver transplantation, surgical resection or local ablation in BCLC stage 0 and stage $A$, trans arterial chemo embolisation in BCLC stage $B$, sorafenib in stage $C$ and symptomatic therapy in stage D. It was then analysed whether stage appropriate management was administered. Factors if any hindering the stage appropriate management was looked into. Cirrhosis was diagnosed based on standard criteria. Patients lost to follow up were contacted telephonically in March 2015.

\section{Methods of Data Collection}

Investigator who collected data was not involved in patient care. Names of the patients were coded, and anonymity was maintained. Nonprobability sampling of all patients during the study was conducted. Selection bias was minimised by nonselective inclusion of all patients during the study period. Checklist using stage specific BCLC management strategies was undertaken. 


\section{Statistical Analysis}

Statistical analysis was done using IBM SPSS software version 24. Simple percentages were graphed against various parameters and reports were analysed.

\section{RESULTS}

A total of 32 patients [males 29 (90.6\%), mean age $58.75 \pm$ 12.47 years] of HCC were included. In three fourth of the patients HCC was diagnosed based on typical findings on dynamic imaging studies (triple phase contrast enhanced CT abdomen and / or MRI abdomen). Liver biopsy was needed in one fourth of the patients. ${ }^{8}$ Majority of the patients $(87.5 \%)$ had cirrhosis of the liver at the time of diagnosis of HCC. Some of these patients [5 (17.8\%)] were known cirrhotic patients. Majority of the known cirrhotics [3 (10.7\%)] were regular with their outpatient visits. HCC could be diagnosed at a relatively earlier stage in these patients. Serum alpha fetoprotein (AFP) was $>40 \mathrm{ng}$ in fourteen patients (43.75\%). Portal vein thrombosis was seen in 4 patients at the time of diagnosis while distant metastases was seen in 3 patients. Most common cause of HCC was hepatitis B virus (HBV) seen in 21 patients $(65.625 \%)$. Only two of all the patients with HBV related HCC were non cirrhotic. Six of all the patients with HBV associated HCC were HBV treatment naïve. Among the fifteen patients on antivirals for hepatitis B, six were on regular follow up. Both the patients with hepatitis $C$ virus (HCV) associated HCC were of genotype 3 and were cirrhotics. Among all the HCV genotypes, genotype 3 is associated with higher treatment failures, disease recurrence and higher rate of complications like fatty liver, liver fibrosis and HCC development. ${ }^{9}$ One of the patient with HCV associated HCC had completed treatment for HCV 3 years back and had achieved sustained virologic response (SVR) 12 before dropping out of regular outpatient visits and HCC surveillance. Alcoholic liver disease was responsible for 6 of the 32 patients. One among them was noncirrhotic. Stage appropriate treatment could not be given to $50 \%$ of patients in BCLC stage B due to financial constraints. $65.22 \%$ of patients in BCLC stage C were started on sorafenib (15 out of 23 patients). Drug dose had to be reduced in 5 of the 15 patients (33\%) while it had to be discontinued in 3 patients ( $20 \%$ ). All patients of BCLC stage D received stage appropriate therapy. Mean survival at 1 year was 238, 70 and 34.5 days in BCLC stages B, C and D respectively.

$79.3 \%$ patients were in BCLC stage C, while $17.2 \%$ were in BCLC stage D and $13.8 \%$ were BCLC stage B. $43.47 \%$ of patients in BCLC stage $C$ progressed while $50 \%$ of patients in BCLC stage B progressed to advanced stage during the study.

HBV was the leading cause responsible for $65.625 \%$ of cases followed by alcoholic liver disease (18.75 \% of all cases). HCV caused $6.25 \%$ of all cases. Other causes included nonalcoholic fatty disease or metabolic associated fatty liver disease. $50 \%$ of patients in BCLC stage B received stage appropriate treatment while $65.22 \%$ of all patients in BCLC stage $\mathrm{C}$ and $100 \%$ of patients in BCLC stage D received stage appropriate treatment. $62.5 \%$ of close contacts of HBV related HCC patients were not vaccinated. Only $26.66 \%$ of close contacts had received vaccine for HBV. Of the contacts who had received vaccine for HBV only $25 \%$ knew their immunisation status. $6.66 \%$ of close contacts were found to be HBsAg positive on testing.
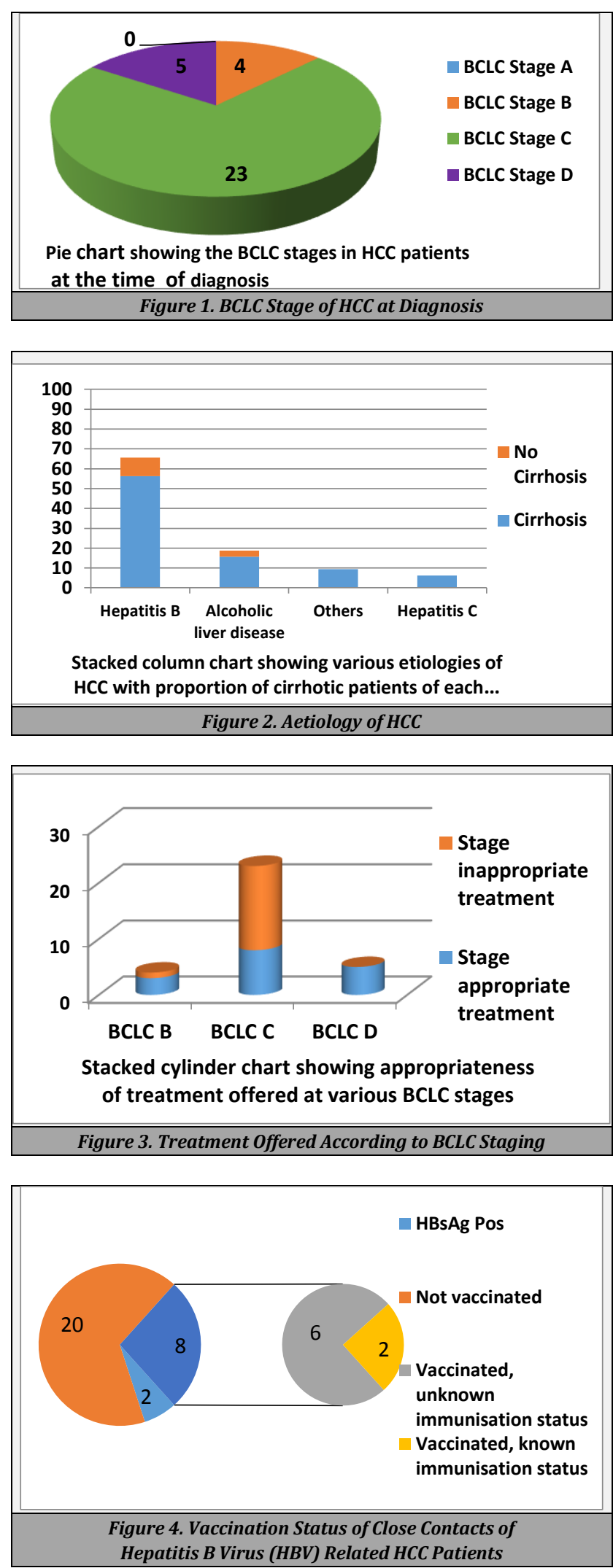

\section{DISCUSSION}

Cirrhosis of liver is a well-known high-risk condition for development of HCC. About $80 \%$ of HCC has been reported in 
patients with cirrhosis in the entire world. ${ }^{10}$ Most of the HCC cases in the developed countries are diagnosed early due to availability of better surveillance strategies. However, in our country, most of the patients are diagnosed in advanced stages which results in high mortality rates. Indian data on HCC shows it to be the seventh most common cause of cancer related death in India. ${ }^{11}$ As per reports from tertiary care centres in India, 70 - $97 \%$ of patients with HCC had underlying liver cirrhosis at the time of diagnosis of HCC.12,13 HCC is extremely common in areas endemic for hepatitis $\mathrm{B}$ and $\mathrm{C}$ virus. Although India is an endemic zone for hepatitis B virus, there has not been comprehensively analysed data for HCC and cancer registries probably do not reflect the accurate incidence. ${ }^{14}$ In our country, $70 \%-80 \%$ of all HCCs are attributed to $\mathrm{HBV}$, approximately $15 \%$ are attributed to hepatitis $\mathrm{C}$ virus and $5 \%$ are attributed to both HBV and HCV. ${ }^{15}$ Alcohol was responsible for about for $8 \%$ of all HCCs, while in about $10 \%$, no direct aetiology was seen. In our study HBV was responsible for $65.625 \%$ of cases followed by alcoholic liver disease (18.75 \% of all cases) and HCV (6.25\% of all cases). HBV is a vaccine preventable disease. In Taiwan the relative risk of HCC in 6 to 26-year olds was significantly lower among the cohort after implementation of universal hepatitis $B$ vaccination than in the before cohort. ${ }^{16}$ In India universal hepatitis B vaccination program was extended to cover the entire nation in 2012. In a nationally representative serosurvey conducted during 2017 to assess the impact of universal vaccination against HBV it was found that children born after the introduction of hepatitis $B$ vaccination had a lower prevalence of past HBV infection and a higher prevalence of anti HBs in statistically significant proportions. ${ }^{17}$ In our study only $26.66 \%$ of close contacts had received vaccine for HBV. Of the contacts who had received vaccine for HBV only $25 \%$ knew their immunisation status.

There are four large case series reporting HCC from India which comprise a total of 952 cases presenting to various tertiary centres showing similar results with respect to aetiology of HCC.18,19,20,21

\section{CONCLUSIONS}

Most of HCC patients have underlying cirrhosis at the time of diagnosis. Hepatitis B is the most common aetiology which is vaccine preventable. Surveillance recommendations must be strictly adhered so that HCC can be diagnosed at an early stage. Prevention of HBV infection with vaccine should be done in close contacts on time. However, affordability and compliance will remain a major issue of HCC management in our country. Cheaper treatment alternatives for HCC are needed.

Data sharing statement provided by the authors is available with the full text of this article at jemds.com.

Financial or other competing interests: None.

Disclosure forms provided by the authors are available with the full text of this article at jemds.com.

\section{REFERENCES}

[1] The Cancer of the Liver Italian Program (CLIP) investigators. A new prognostic system for hepatocellular carcinoma: a retrospective study of 435 patients. Hepatology 1998;28(3):751-5.

[2] Marrero JA, Fonatana RJ, Barrat A, et al. Prognosis of hepatocellular carcinoma: comparison of 7 staging systems in an American cohort. Hepatology 2005;41(4):707-16.

[3] Llovet JM, Fuster J, Bruix J, et al. The barcelona approach: diagnosis, staging, and treatment of hepatocellular carcinoma. Liver Transplantation 2004;10(2 Suppl 1):S115-S20.

[4] European Association for the Study of The Liver, European Organisation for Research and Treatment of Cancer. EASL - EORTC clinical practice guidelines: management of hepatocellular carcinoma. Journal of Hepatology 2012;56(4):908-43.

[5] Saito A, Toyoda H, Kobayashi M, et al. Prediction of early recurrence of hepatocellular carcinoma after resection using digital pathology images assessed by machine learning. Modern Pathology 2021;34(2):417-25.

[6] European Association for the Study of the Liver. EASL clinical practice guidelines: management of hepatocellular carcinoma. Journal of Hepatology 2018;69(1):182-236.

[7] Mehta N, Yao FY. What are the optimal liver transplantation criteria for hepatocellular carcinoma? Clinical Liver Disease 2019;13(1):20-5.

[8] Quinto AM, Quana-Anisa N, Manso RSR, et al. Complications of Transarterial Chemoembolization (TACE) in the treatment of liver tumors. Cirugia Espanola 2018;96(9):560-7.

[9] Shahnazarian V, Ramai D, Reddy M, et al. Hepatitis C virus genotype 3: clinical features, current and emerging viral inhibitors, future challenges. Annals of Gastroenterology 2018;31(5):541-51.

[10] Lokesh KN, Chaudhuri Tamojit, Lakshmaiah KC, et al. Advanced hepatocellular carcinoma: a regional cancer center experience of 48 cases. Indian Journal of Cancer 2017;54(3):526-9.

[11] Acharya SK. Epidemiology of hepatocellular carcinoma in India. J Clin Exp Hepatol 2014;4(Suppl 3):S27-S33.

[12] Sarin SK, Thakur V, Guptan RC, et al. Profile of hepatocellular carcinoma in India: an insight into the possible etiologic associations. J Gastroenterol Hepatol 2001;16(6):666-73.

[13] Paul SB, Chalamalasetty SB, Vishnubatla S, et al. Clinical profile, etiology and therapeutic outcome in 324 hepatocellular carcinoma patients at a tertiary care center in India. Oncology 2009;77(3-4):162-71.

[14] Mallath MK, Taylor DG, Badwe RA, et al. The growing burden of cancer in India: epidemiology and social context. Lancet Oncol 2014;15(6):e205-12.

[15] Kumar R, Saraswat MK, Sharma BC, et al. Characteristics of hepatocellular carcinoma in India: a retrospective analysis of 191 cases. QJM 2008;101(6):479-85. 
[16] Chang MH, You SL, Chen CJ, et al. Long - term effects of Hepatitis B immunization of infants in preventing liver cancer. Gastroenterology 2016;151(3):472-80.e1.

[17] Murhekar MV, Kumar MS, Kamaraj P, et al. Hepatitis - B virus infection in India: findings from a nationally representative serosurvey, 2017-18. International Journal of Infectious Diseases 2020;100:455-60.

[18] Ashim M, Sarma MP, Kar P. Etiological and molecular profile of hepatocellular cancer from India. Int J Cancer 2013;133(2):437-45.
[19] Sharma S, Sharma B, Chawla YK, et al. Comparison of 7 staging system in north Indian cohort of hepatocellular carcinoma. Trop Gastroenterol 2010;31(4):271-8.

[20] Mohandas KM. Surveillance of Indians with liver cirrhosis for treatable hepatocellular carcinoma: another enigma. Indian J Gastroenterol 2007;26(6):261-4.

[21] Tanaka K, Sakai H, Hashizume M, et al. A long - term follow-up study on risk factors for hepatocellular carcinoma among Japanese patients with liver cirrhosis. Jpn J Cancer Res 1998;89(12):1241-50. 